\title{
PDZRN3 regulates the differentiation of myoblasts into myotubes through Id2- mediated pathway
}

\author{
Takeshi Honda, Narumi Nakada, Ryoto Nagai, Yuki Yokosuka, Makoto Inui \\ Department of Pharmacology, Yamaguchi University Graduate School of Medicine, Japan
}

[Background] PDZRN3 is a member of the PDZ domain-containing RING finger protein family and is involved in a variety of developmental processes. We first cloned PDZRN3 cDNA from a human heart library. PDZRN3 is also expressed in skeletal muscle and is essential for myogenic differentiation. Depletion of PDZRN3 by RNAi inhibits differentiation of $\mathrm{C} 2 \mathrm{C} 12$ myoblasts into myotubes, suppressing expression of myosin heavy chain (MHC). However, the depletion of PDZRN3 unaffected the expression of a muscle specific basic-helix-loop-helix transcription factor (bHLH), myogenin, which induces the MHC expression. Here we investigated how PDZRN3 regulates the MHC expression and myogenic differentiation.

[Methods and Results] The luciferase reporter assay revealed that the MHC-promoter activity decreased in PDZRN3depleted cells, suggesting that the transcriptional activity of myogenin is inhibited without a change of myogenin expression. We therefore examined the effects of PDZRN3-depletion on expression of Id2, a dominant negative regulator of bHLH. It has been known that Id2 rapidly decreased after induction of differentiation, making myogenin active. PDZRN3-depletion significantly delayed the down-regulation of Id2, resulting in a prolonged inhibition of the transcriptional activity of myogenin. On the other hand, the suppression of Id2 by RNAi restored the MHC expression in PDZRN3-depleted cells, removing the Id2 inhibition of myogenin. The Id2-promoter assay revealed that STAT5b was a factor enhancing the Id2 expression in PDZRN3-depleted cells. We also found that PDZRN3-depletion transcriptionally up-regulated the STAT5b expression. The overexpression of STAT5b increased the amounts of Id2 protein in C2C12 cells and suppressed the induction of MHC. Furthermore, depletion of PDZRN3 decreased the phosphorylated form of $\mathrm{Id} 2$ and concomitantly increased the unphosphorylated form in $\mathrm{C} 2 \mathrm{C} 12$ cells. It has been known that the Id2 inhibition of myogenin is relieved by phosphorylation of Id2 catalyzed by Cdk2 with cyclin A2. The expression of cyclin A2 was significantly decreased in PDZRN3-depleted cells, suggesting that the enhanced Id2 inhibition of myogenin in PDZRN3depleted cells might be partly due to the decrease of the phosphorylated form of Id2.

[Conclusion] These results indicate that PDZRN3 plays a crucial role in differentiation of myoblasts into myotubes by regulating Id2 transcriptionally as well as post-translationally. 